\title{
HOPF ALGEBRAS WITH NONSEMISIMPLE ANTIPODE
}

\author{
EARL J. TAFT ${ }^{1}$ AND ROBERT LEE WILSON ${ }^{2}$
}

ABSTRACT. An example is given to show that the antipode of a finite dimensional Hopf algebra over a field of prime characteristic $p>2$ need not be semisimple. (For $p=2$ examples were previously known.) The example is a pointed irreducible Hopf algebra $H$ (with antipode $S$ ) of dimension $p^{3}$ such that $S^{2 p}=I \neq S^{2}$.

Radford [4] has recently shown that the antipode $S$ of a finite dimensional Hopf algebra $H$ over a field $K$ has finite order. Consequently, if $K$ is of characteristic zero then the antipode of $H$ is semisimple. On the other hand, if $K$ is of characteristic 2 then $S$ is semisimple only if $S=I$. (For otherwise $S$ has even order, say $2 k$, and so $0=S^{2 k}-I=\left(S^{k}-I\right)^{2}$.) In this note we show that $S$ may fail to be semisimple for any characteristic $p \geq 3$. We do this by constructing a pointed irreducible Hopf algebra of dimension $p^{3}$ over an arbitrary field of characteristic $p \geq 3$ in which the antipode has order $2 p$ (and hence is not semisimple).

A related problem is that of finding a bound for the order of $S$. In [7] the authors have shown that if $H$ is pointed, if $G(H)$ has exponent $e$ and if $H_{0}$ $\subseteq H_{1} \subseteq \cdots \subseteq H_{m}=H$ is the coradical filtration then $\left(S^{2 e}-I\right)^{m}=0$. Thus if $K$ has characteristic $p$ and $p^{n-1}<m \leq p^{n}$ then $S^{2 e p^{n}}=I$. Thus the order of $S$ divides $2 e p^{n}$. A number of finite dimensional pointed Hopf algebras are known [2], [3], [6] in which the order of the antipode is $2 e$. (In one of these, due independently to Radford [3] and Sweedler (described in [2]), $n=0$ and hence the upper bound $2 e p^{n}$ is actually attained.) The example given here is the first of a pointed Hopf algebra in which the order of $S$ exceeds $2 e$ (here $e=1)$. Whether the order of the antipode can actually equal $2 e p^{n}$ when $n \geq 1$ remains an open question.

Presented to the Society, January 17, 1974 under the title Some finite dimensional pointed Hopf algebras with nonsemisimple antipode; received by the editors October 25, 1973.

AMS (MOS) subject classifications (1970). Primary 16A24.

${ }^{1}$ This research was partially supported by National Science Foundation grant GP-38518.

2 This research was partially supported by National Science Foundation grant GP-33226. 
1. Definition of $H$. Let $K$ be a field of characteristic $p \geq 3$ and let $R$ denote the free algebra over $K$ on three noncommuting variables $X, Y$, and $Z$. Since $R$ is free there is a unique homomorphism $\Delta: R \rightarrow R \otimes R$ such that

$$
\begin{gathered}
\Delta(X)=1 \otimes X+X \otimes 1, \Delta(Y)=1 \otimes Y+Y \otimes 1, \\
\Delta(Z)=1 \otimes Z+Z \otimes 1+X \otimes Y .
\end{gathered}
$$

It is easily checked that $(I \otimes \Delta) \Delta$ and $(\Delta \otimes I) \Delta$ agree on the free generators $X, Y$, and $Z$ and hence that $\Delta$ is coassociative. We define an algebra homomorphism $\epsilon: R \rightarrow K$ by $\epsilon(X)=\epsilon(Y)=\epsilon(Z)=0$. It is immediate that $(I \otimes \epsilon) \Delta$ $=(\epsilon \otimes I) \Delta=I$. Hence $R$ is a bialgebra.

Let $\mathcal{G}$ be the ideal in $R$ generated by $[X, Y]-X,[Y, Z]+Z,[X, Z]-$ $X^{2} / 2, X^{p}, Y^{p}-Y$, and $Z^{p}$.

We wish to show that $g$ is a bi-ideal, i.e., that $\Delta(\mathscr{g}) \subseteq R \otimes \mathscr{I}+9 \otimes R$ (obviously $\epsilon(\mathcal{G})=(0)$ ). It is sufficient to check this on generators for $\mathscr{I}$. For the generators $[X, Y]-X, X^{p}$, and $Y^{p}-Y$ the result follows from the fact that in a bialgebra over a field of characteristic $p$ the primitive elements form a restricted Lie algebra. For the remaining generators we compute:

$$
\begin{aligned}
& \Delta([Y, Z]+Z)=[\Delta Y, \Delta Z]+\Delta Z \\
& =[1 \otimes Y+Y \otimes 1,1 \otimes Z+Z \otimes 1+X \otimes Y] \\
& +1 \otimes Z+Z \otimes 1+X \otimes Y \\
& =1 \otimes[Y, Z]+[Y, Z] \otimes 1+[Y, X] \otimes Y \\
& +1 \otimes Z+Z \otimes 1+X \otimes Y \\
& =1 \otimes([Y, Z]+Z)+([Y, Z]+Z) \otimes 1-([X, Y]-X) \otimes Y \\
& \Delta\left([X, Z]-X^{2} / 2\right)=[\Delta X, \Delta Z]-(\Delta X)^{2} / 2 \\
& =[1 \otimes X+X \otimes 1,1 \otimes Z+Z \otimes 1+X \otimes Y] \\
& -\left(X^{2} / 2\right) \otimes 1-X \otimes X-1 \otimes\left(X^{2} / 2\right) \\
& =1 \otimes[X, Z]+[X, Z] \otimes 1+X \otimes[X, Y] \\
& -\left(X^{2} / 2\right) \otimes 1-X \otimes X-1 \otimes\left(X^{2} / 2\right) \\
& =1 \otimes\left([X, Z]-\left(X^{2} / 2\right)\right)+X \otimes([X, Y]-X) \\
& +\left([X, Z]-\left(X^{2} / 2\right)\right) \otimes 1 ; \\
& \Delta\left(Z^{p}\right)=(\Delta Z)^{p}=(1 \otimes Z+Z \otimes 1+X \otimes Y)^{p} .
\end{aligned}
$$


Jacobs on [1, formula (63), p. 187] has shown that if $a$ and $b$ are elements of an associative algebra over a field of characteristic $p$ then

$$
(a+b)^{p}=a^{p}+b^{p}+\sum_{i=1}^{p-1} s_{i}
$$

Hence

$$
\text { where is }{ }_{i} \text { is the coefficient of } \lambda^{i-1} \text { in } a(\operatorname{ad}(\lambda a+b))^{p-1} \text {. }
$$

$$
\Delta\left(Z^{p}\right)=(1 \otimes Z)^{p}+(Z \otimes 1+X \otimes Y)^{p}+\sum_{i=1}^{p-1} s_{i}
$$

where is ${ }_{i}$ is the coefficient of $\lambda^{i-1}$ in

$$
(1 \otimes Z)(\operatorname{ad}(\lambda(1 \otimes Z)+Z \otimes 1+X \otimes Y))^{p-1} .
$$

Now

$$
[1 \otimes Z, \lambda(1 \otimes Z)+Z \otimes 1+X \otimes Y]=X \otimes[Z, Y] \equiv X \otimes Z
$$

(where all congruences are modulo $9 \otimes R+R \otimes 9$ ). Now since $[X, Z] \equiv X^{2} / 2$ (and $[a b, c]=a[b, c]+[a, c] b$ for any elements $a, b$, and $c$ in an associative algebra) we have $\left[X^{n}, Z\right] \equiv(n / 2) X^{n+1}$. Hence

$$
\begin{aligned}
{\left[X^{i} \otimes Z, \lambda(1 \otimes Z)+(Z \otimes 1+X \otimes Y)\right] } & =\left[X^{i}, Z\right] \otimes Z+X^{i+1} \otimes[Z, Y] \\
& \equiv((i+2) / 2) X^{i+1} \otimes Z .
\end{aligned}
$$

Thus by induction we see that

$$
(1 \otimes Z)(\operatorname{ad}(\lambda(1 \otimes Z)+Z \otimes 1+X \otimes Y))^{i} \equiv\left((i+1) ! / 2^{i}\right) X^{i} \otimes Z
$$

and so

$$
(1 \otimes Z)(\operatorname{ad}(\lambda(1 \otimes Z)+Z \otimes 1+X \otimes Y))^{p-1} \equiv 0
$$

Hence we have

$$
\Delta\left(Z^{p}\right) \equiv 1 \otimes Z^{p}+(Z \otimes 1+X \otimes Y)^{p} .
$$

Using (*) again we see that

$$
(Z \otimes 1+X \otimes Y)^{p}=(Z \otimes 1)^{p}+(X \otimes Y)^{p}+\sum_{i=1}^{p-1} t_{i}
$$

where $i t_{i}$ is the coefficient of $\lambda^{i-1}$ in

$$
(Z \otimes 1)(\operatorname{ad}(\lambda(Z \otimes 1)+X \otimes Y))^{p-1}
$$

Now

$$
[Z \otimes 1, \lambda(Z \otimes 1)+X \otimes Y]=[Z, X] \otimes Y \equiv-1 / 2 X^{2} \otimes Y
$$


Furthermore,

$$
\left[X^{i} \otimes Y, \lambda(Z \otimes 1)+X \otimes Y\right]=\lambda\left[X^{i}, Z\right] \otimes Y \equiv(\lambda i / 2) X^{i+1} \otimes Y .
$$

It follows by induction that $(Z \otimes 1)(\operatorname{ad}(\lambda(Z \otimes 1)+X \otimes Y))^{i}$ is congruent to a multiple of $X^{i+1} \otimes Z$ and hence that $(Z \otimes 1)(\operatorname{ad}(\lambda(Z \otimes 1)+X \otimes Y))^{p-1} \equiv 0$. Hence $\Delta\left(Z^{p}\right) \equiv 1 \otimes Z^{p}+Z^{p} \otimes 1$. This completes the proof that $g$ is a biideal.

Let $S$ be the unique antihomomorphism of $R$ such that

$$
S(X)=-X, \quad S(Y)=-Y, \quad S(Z)=X Y-Z .
$$

We claim that $S(\mathscr{g}) \subseteq 9$. It is sufficient to check this on generators. We do this as follows (where all congruences are modulo $g$ ):

$$
\begin{aligned}
S([X, Y]-X) & =[S(Y), S(X)]-S(X)=[-Y,-X]+X=-([X, Y]-X) \\
S([Y, Z]+Z) & =[S(Z), S(Y)]+S(Z)=[X Y-Z,-Y]+X Y-Z \\
& =-[X, Y] Y-[Y, Z]+X Y-Z \equiv-([Y, Z]+Z) \\
S\left([X, Z]-X^{2} / 2\right) & =[S(Z), S(X)]-S(X)^{2} / 2=[X Y-Z,-X]-X^{2} / 2 \\
& =X[X, Y]+[Z, X]-X^{2} / 2 \equiv-\left([X, Z]-X^{2} / 2\right) ; \\
S\left(X^{p}\right) & =(S(X))^{p}=-X^{p} ; \\
S\left(Y^{p}-Y\right) & =(S(Y))^{p}-S(Y)=-\left(Y^{p}-Y\right) ; \\
S\left(Z^{p}\right) & =(S(Z))^{p}=(X Y-Z)^{p}=(X Y)^{p}-Z^{p}+\sum_{i=1}^{p-1} u_{i}
\end{aligned}
$$

where $i u_{i}$ is the coefficient of $\lambda^{i-1}$ in $(X Y)(\operatorname{ad}(\lambda X Y-Z))^{p-1}$ (again by $\left.(*)\right)$. Now as $[X, Y] \equiv X$ it is immediate that $(X Y)^{p} \equiv 0$. Also $Z^{p} \equiv 0$. Now $[X, X Y]$ $=X[X, Y] \equiv X^{2}$ and so $\left[X^{i}, X Y\right] \equiv i X^{i+1}$. Also $\left[X^{i}, Z\right] \equiv(i / 2) X^{i+1}$. Thus $\left[X^{i}, \lambda X Y-Z\right] \equiv i(\lambda-1 / 2) X^{i+1}$. Furthermore,

$$
\begin{aligned}
{[(X Y / 2)-Z, \lambda X Y-Z] } & =(\lambda-1 / 2)[X Y, Z]=(\lambda-1 / 2)([X, Z] Y+X[Y, Z]) \\
& \equiv(\lambda-1 / 2) X((X Y / 2)-Z) .
\end{aligned}
$$

Thus

$$
\begin{aligned}
{\left[X^{i}((X Y / 2)-Z), \lambda X Y-Z\right] } & =\left[X^{i}, \lambda X Y-Z\right]((X Y / 2)-Z)+X^{i}[(X Y / 2)-Z, \lambda X Y-Z] \\
& \equiv(i+1)(\lambda-1 / 2) X^{i+1}((X Y / 2)-Z) .
\end{aligned}
$$

Now since $[X Y, \lambda X Y-Z]=-[X Y, Z] \equiv-X((X Y / 2)-Z)$ it follows by induc- 
tion that

$$
(X Y)(\operatorname{ad}(\lambda X Y-Z))^{i} \equiv-i !(\lambda-1 / 2)^{i-1} X^{i}((X Y / 2)-Z)
$$

and so

$$
\begin{aligned}
(X Y)(\operatorname{ad}(\lambda X Y-Z))^{p-1} & \equiv-(p-1) !(\lambda-1 / 2)^{p-2} X^{p-1}((X Y / 2)-Z) \\
& \equiv-(\lambda-1 / 2)^{p-2} X^{p-1} Z .
\end{aligned}
$$

But then

$$
\sum_{i=1}^{p-1} u_{i} \equiv\left(-\int_{0}^{1}(\lambda-1 / 2)^{p-2} d \lambda\right) X^{p-1} Z=0
$$

Hence $S\left(Z^{p}\right) \equiv 0$, as required.

Now let $H=R / 9$ and let $x=X+9, y=Y+9$, and $z=Z+9$. As 9 is a bi-ideal, $H$ is a bialgebra. We denote the coalgebra structure maps in $H$ by $\Delta$ and $\epsilon$. Also, since $S(\mathscr{g}) \subseteq 9, S$ induces an antihomomorphism of $H$, which we again denote by $S$. Then $S(x)=-x, S(y)=-y$, and $S(z)=x y-z$. We claim that $H$ is a Hopf algebra with antipode $S$. To verify this we must check that $m(S \otimes I) \boldsymbol{\Delta}=m(I \otimes S) \boldsymbol{\Delta}=\mu \epsilon$ (where $m$ and $\mu$ are the algebra structure maps for $H$ ). Now it is sufficient to check this on generators for $H$. For $x$ and $y$ this is immediate, and for $z$ we have

$$
m(S \otimes I) \Lambda(z)=m(I \otimes S) \Lambda(z)=S(z)+z-x y=0=\mu \epsilon(z),
$$

as required.

2. A basis for $H$. It is clear that $H$ is spanned by $\left\{x^{i} y^{j} z^{k} \mid 0 \leq i, j, k \leq p-1\right\}$. We will now show that this is a basis for $H$.

Consider the following $p$ by $p$ matrices over $K$ (where $E_{i j}$ denotes the usual matrix unit):

$$
A_{i}=E_{p-i, p}, \quad 1 \leq i \leq p-1, \quad B=\sum_{i=1}^{p}(i+1) E_{i i},
$$

and

$$
C=\sum_{i=1}^{p-1}((i+1) / 2) E_{i, i+1}
$$

It is easily checked that $\left[A_{i}, A_{j}\right]=0$ for $1 \leq i, j \leq p-1,\left[A_{i}, B\right]=i A_{i}$ for $1 \leq i \leq p-1,\left[A_{i}, C\right]=(i / 2) A_{i+1}$ for $1 \leq i \leq p-2,\left[A_{p-1}, C\right]=0,[B, C]=-C$, $A_{i}^{p}=0$ for $1 \leq i \leq p-1, B^{p}=B$, and $C^{p}=0$. Thus $\left\{A_{i} \mid 1 \leq i \leq p-1\right\} \cup$ $\{B\} \cup\{C\}$ forms a basis for a restricted Lie algebra. Denote this algebra by 
$\mathcal{B}$ and its restricted enveloping algebra by $\mathcal{U}$.

Let $I$ denote the linear span of the set $\left\{A_{i}-A_{1}^{i} \mid 2 \leq i \leq p-1\right\}$ of elements of $\mathcal{U}$. It is easily seen that $[\mathcal{G}, \mathfrak{B}] \subseteq \mathcal{G}$. (The only nontrivial verifications are

$$
\begin{aligned}
{\left[A_{i}-A_{1}^{i}, C\right] } & =(i / 2)\left(A_{i+1}-A_{1}^{i-1} A_{2}\right) \\
& =(i / 2)\left(\left(A_{i+1}-A_{1}^{i+1}\right)-\left(A_{2}-A_{1}^{2}\right) A_{1}^{i-1}\right) \quad \text { for } 2 \leq i<p-1
\end{aligned}
$$

and

$$
\left.\left[A_{p-1}-A_{1}^{p-1}, C\right]=-1 / 2 A_{1}^{p-2} A_{2}=1 / 2\left(A_{2}-A_{1}^{2}\right) A_{1}^{p-2} .\right)
$$

Since $\mathfrak{B}$ generates $\mathcal{U}$ this shows that $\mathcal{U} \mathcal{G} U=\mathscr{J U}$. Let $\mathbb{Q}=\mathcal{U} /(\mathfrak{G})$. Let $A_{1}+$ $g \mathcal{U}=a, B+g \mathcal{U}=b$, and $C+g \mathcal{U}=c$. Clearly $\left\{a^{i} b^{j} c^{k} \mid 0 \leq i, j, k \leq p-1\right\}$ spans A. We wish to show that this set is linearly independent. To this end assume

$$
\sum_{i, j, k} \alpha_{i j k} a^{i} b^{j} c^{k}=0
$$

where $\alpha_{i j k} \in K$. Then

$$
\sum_{i, j, k} \alpha_{i j k} A_{1}^{i} B^{j} C^{k} \in \mathcal{G U}
$$

Since

$$
\left\{A_{1}^{i}{ }_{1} \cdots A_{p-1}^{i}{ }_{p-1} B^{i} C^{k} \mid 0 \leq i_{1}, \cdots, i_{p-1}, j, k \leq p-1\right\}
$$

is a basis for $\mathrm{U}$ it follows that for each $j$ and $k, \Sigma_{i} \alpha_{i j k} A_{1}^{i} \in \mathcal{J O}$ where $\mathcal{O}$ is the linear span of

$$
\left\{A_{1}^{i} \cdots A_{p-1}^{i p-1} \mid 0 \leq i_{1}, \cdots, i_{p-1} \leq p-1\right\}
$$

Define a homomorphism $\phi: \vartheta \rightarrow \circlearrowright$ by

$$
\phi\left(A_{1}^{i} \cdots A_{p-1}^{i}{ }_{p-1}\right)=A_{1}^{i_{1}+2 i_{2}+\cdots+(p-1) i_{p-1}}
$$

Note that $\mathcal{I}$ and hence $\mathcal{G O}$ are contained in $\operatorname{ker} \phi$. On the other hand $\phi\left(\Sigma_{i} a_{i j k} A_{1}^{i}\right)=\Sigma_{i} a_{i j k} A_{1}^{i}$. Thus $\Sigma_{i} \alpha_{i j k} A_{1}^{i} \in \mathcal{I O}$ implies $\Sigma_{i} \alpha_{i j k} A_{1}^{i}=0$ so $a_{i j k}=0$ for all $i, j$, and $k$, as required. Thus $\mathcal{Q}$ is of dimension $p^{3}$.

Now define a homomorphism $\psi: R \rightarrow \mathfrak{A}$ by $\psi(X)=a, \psi(Y)=b$, and $\psi(Z)=c$. Then ker $\psi \supseteq \underline{9}^{\text {so } \psi} \psi$ induces a homomorphism of $H$ onto $\mathbb{Q}$. Hence $\operatorname{dim} H \geq p^{3}$. Since we already have that $\left\{x^{i} y^{j} z^{k} \mid 0 \leq i, j, k \leq p-1\right\}$ spans $H$ this shows that $\left\{x^{i} y^{j} z^{k} \mid 0 \leq i, j, k \leq p-1\right\}$ is a basis for $H$. 
3. Properties of $H$. We will now show that $H$ is pointed and irreducible and that the antipode of $H$ has order $2 p$.

Lemma 1. If $C$ is a coalgebra and $W$ is a simple subcoalgebra, then $\bigwedge^{n} W$ is an irreducible subcoalgébra of $C$.

Proof. That $\bigwedge^{n} W$ is a subcoalgebra follows from Proposition 9.0.0(i) of [5]. We prove irreducibility by induction on $n$. Since $\wedge^{1} W=W$ is simple the result holds for $n=1$. Assume the result for $n-1$. Let $Y$ be a simple subcoalgebra of $\bigwedge^{n} W$. Then

$$
\begin{aligned}
(0) & \neq \Delta Y \subseteq(Y \otimes Y) \cap\left(C \otimes \wedge^{n-1} W+W \otimes C\right) \\
& \subseteq(Y \otimes Y) \cap\left(C \otimes \wedge^{n-1} W+\wedge^{n-1} W \otimes C\right) .
\end{aligned}
$$

It then follows that $Y \cap \wedge^{n-1} W \neq(0)$ and so, since $Y$ is simple, $Y \subseteq$ $\bigwedge^{n-1} W$. Then by the induction assumption $Y=W$.

Lemma 2. If $M$ is a bialgebra and $W$ is a subbialgebra then

$$
\left(\bigwedge^{m} W\right)\left(\bigwedge^{n} W\right) \subseteq \bigwedge^{m+n-1} W
$$

for all $m$ and $n \geq 1$.

Proof. Since multiplication is a coalgebra map this follows from Lemmas 9.1.3 and 9.2.1 of [5].

Corollary. $H$ is a pointed irreducible Hopf algebra.

Proof. Take $W=K$. Then $W$ is a subbialgebra and a simple coalgebra. Now $x$ and $y \in \wedge^{2} W$ and $z \in \Lambda^{3} W$. Then since $\{x, y, z\}$ generates $H$, Lemma 2 shows that $H=\bigwedge^{n} W$ for some $n$. Hence by Lemma $1 H$ is irreducible (and is pointed since $W$ is pointed). We have already show $n$ that $H$ is a Hopf algebra with antipode $S$ (although we could have avoided doing this, since by Theorem 9.2.2 of [5] every irreducible bialgebra is a Hopf algebra).

It remains only to determine the order of $S$. Now

$$
S^{2}(z)=S(y) S(x)-S(z)=y x-x y+z=[y, x]+z=z-x .
$$

Then $S^{2 i}(z)=z-i x$ for all $i$. Hence $S$ has order $2 p$.

We summarize our results in the following

Proposition. $H$ is a pointed irreducible Hopf algebra of dimension $p^{3}$. The antipode of $H$ has order $2 p$.

4. A property of $H^{*}$. Let $J$ be the ideal of $H$ generated by $x$ and $z$. Then $J$ is a nilpotent ideal in $H$. (For when a monomial is straightened its 
total degree in $x$ and $z$ is preserved.) The quotient $H / J$ has basis $\left\{y^{j}+J \mid 0 \leq j \leq p-1\right\}$ and is hence isomorphic to $K[t] /\left(t^{p}-t\right)$, i.e., it is isomorphic to a direct product of $p$ copies of $K$. It follows that $J=\operatorname{Rad} H$, that $H^{*}$ is pointed, and that $G\left(H^{*}\right)$ has order $p$. Thus order of $S^{*}=$ order of $S=$ $2 p=2\left(\right.$ exponent of $\left.G\left(H^{*}\right)\right)$.

Whether or not there exists a finite dimensional pointed Hopf algebra $H$ with $H^{*}$ pointed, order $S>2$ (exponent of $G(H)$ ), and order $S>2$ (exponent of $\left.G\left(H^{*}\right)\right)$ remains an open question.

\section{REFERENCES}

1. N. Jacobson, Lie algebras, Interscience Tracts in Pure and Appl. Math., no. 10, Interscience, New York, 1962. MR $26 \# 1345$.

2. R. G. Larson, Orders in Hopf algebras, J. Algebra 22 (1972), 201-210. MR 45 \#8706.

3. D. E. Radford, A free rank 4 Hopf algebra with antipode of order 4, Proc. Amer. Math. Soc. 30 (1971), 55-58. MR 43 \#4887.

4. - The order of the antipode of a finite dimensional Hopf algebra is finite, Amer. J. Math. (to appear).

5. M. E. Sweedler, Hopf algebras, Math. Lecture Notes Series, Benjamin, New York, 1969. MR 40 \#5705.

6. E. J. Taft, The order of the antipode of finite-dimensional Hopf algebra, Proc. Nat. Acad. Sci. U.S.A. 68 (1971), 2631-2633. MR 44 \#4075.

7. E. J. Taft and R. L. Wilson, On antipodes in pointed Hopf algebras, J. Algebra 29 (1974), 27-32.

SCHOOL OF MATHEMATICS, INSTITUTE FOR ADVANCED STUDY, PRINCETON, NEW JERSEY 08540

DEPARTMENT OF MATHEMATICS, RUTGERS UNIVERSITY, NEW BRUNSWICK, NEW JERSEY 08903 (Current address of both authors) 